\title{
Fuzzy relational equations and the covering problem
}

\author{
Qing-quan Xiong, Qian-yu Shu \\ College of Mathematics and Software Science, Sichuan Normal University,Chengdu, Sichuan 610066, P. R. China
}

\begin{abstract}
The work considers the problem of solving a system of fuzzy relational equations with inf-implication composition and introduces the concepts of a characteristic matrix and attainable components. It is first shown that solving the system is closely related with the covering problem. Further, it is proved that maximal solutions of the system correspond to irredundant coverings of the characteristic matrix. At last, the necessary and sufficient conditions that the system has a unique(maximal) solution are given.
\end{abstract}

Keywords: Fuzzy relational equation, Maximal solutions, Covering problem, Irredundant covering

\section{Introduction}

Fuzzy relational equations play important roles in many applications, such as intelligence technology [7], compression/decompression of images and videos $[5,19,24]$ and fuzzy decision marking [19], etc. The first paper of fuzzy relational equations was due to Sanchez [25], where the max-min composition was adopted. Recently, there have been many research papers investigating the solvability of fuzzy relational equations $[2,4,7,12,15,16,19-22,27-$ 29]. In fact, fuzzy relational equations can be categorized based on many different compositions. Some common compositions include max-min $[4,7$, $12,25]$, max-product $[7,23]$, max-Archimedean tnorm $[2,4,7,11,17,19]$, inf- $\alpha[7,15,16]$ and inf$\alpha_{\mathcal{T}}[2,7,30]$ compositions. Wu [29] and Di Nola et al. [6] found that in fuzzy relational calculus and reasoning inf- $\alpha$ composition is better. In particular, Di Nola et al. [7] obtained the smallest solution of fuzzy relational equations with inf- $\alpha$ composition and constructed all maximal solutions on a linear lattice when the universe of discourse is finite. $\mathrm{Li}$ and Wang [16] also discussed the fuzzy relational equations with inf- $\alpha$ composition on complete Brouwerian lattices, obtained a necessary and sufficient condition that the solution set is nonempty and described the solution set when the right-hand side of fuzzy relational equations has an irredundant finite meet-decomposition. After that, in finite domains a necessary and sufficient solvability condition was proposed for fuzzy relational equations. Its maximal solutions and the whole solution set were determined, in infinite domains sufficient conditions for existence of a maximal solution for one equation and a system of equations were shown, respectively. Perfilieva [22] considered the problem of solvability for a system of equations with inf- $\rightarrow$ composition on finite universes, where equations were expressed using operations of a BLalgebra. Xiong and Wang [30] dealt with the problem of solving fuzzy relational equations with inf- $\alpha_{\mathcal{T}}$ composition on complete Brouwerian lattices under finite domains (resp. countably infinite domains). When the right-hand sides of fuzzy relational equations are meet-irreducible elements or have irredundant finite meet-decompositions, some necessary and sufficient conditions for attainable solutions (resp. unattainable ones) are formulated and some properties of attainable solutions (resp. unattainable ones) are shown. Chen and Wang [3] provided a proof by transforming polynomially the minimum covering problem, which is a well known NP-hard problem, into the problem of solving a system of sup- $T_{M}$ equations. In the covering problem, the goal is to find all irredundant coverings of a matrix. Markovskii [23] showed that solving fuzzy relational equations with max-product composition is closely related with the covering problem, which belongs to the category of NP-hard problems. Further, Markovskii proved that minimal solutions of system of equations with max-product composition correspond to irredundant coverings. Lin [17] extended Markovskii's work to fuzzy relational equations with max-Archimedean-t-norm composition. Further, Lin [18] investigated fuzzy relational equations with u-norm and transformed the problem of solving a system of fuzzy relational equations into covering problem. In 2012, Shieh [26] developed an efficient algorithm for finding minimal coverings. In fact, Lin $[17,18]$ and Shieh [26] discussed the relations between the minimal solutions of the equations and the irredundant coverings. However, fuzzy relational equations with inf-implication composition have no minimal solutions, there are maximal solutions of the equations, in this paper, we discuss fuzzy relational equations with inf-implication composition on $[0,1]$. In particular, the set of all maximal solutions, and transform the problem of solving fuzzy relational equations into a covering problem. Further, we show that the relations between maximal solutions of fuzzy relational equations with inf-implication and the irredundant coverings of a binary matrix, 
the uniqueness of solution and the covering problem.

This paper is organized as follows. For the sake of convenience, some notions and previous results about the system of inf-implication equations are summarized in Section 2. The concepts of characteristic matrices and attainable components are given and some properties about them are shown in Section 3. The relation between maximal solutions and irredundant coverings is described in Section 4. The necessary and sufficient conditions that the system has a unique(maximal) solution are presented and illustrated in Section 5.

\section{Preliminary}

In the following, unless otherwise stated, let $L=$ $[0,1], I=\{1,2, \ldots, m\}$ and $J=\{1,2, \ldots, n\}$ be two index sets. We are only interested in fuzzy relational equations which have the systemof-equation representation:

$$
A \circ_{\mathcal{I}} X=B
$$

or

$$
\inf _{j \in J} \mathcal{I}\left(a_{i j}, x_{j}\right)=b_{i} \text { for all } i \in I,
$$

where $A=\left(a_{i j}\right)_{I \times J}$ is a matrix with $a_{i j} \in[0,1]$, $B=\left(b_{i}\right)_{i \in I}$ is an $n$-dimensional column vector with $b_{i} \in[0,1]$ for all $i \in I, j \in J$ and $\mathcal{I}$ denotes an implication. The solution set is defined by $\mathscr{X}=$ $\left\{X=\left(x_{j}\right)_{j \in J}: A \circ_{\mathcal{I}} X=B\right\}$. The system (2) is solvable if and only if it has at least one solution.

Definition 2.1 [1,27] (1) A binary operation $\mathcal{T}$ on $L$ is called a pseudo-t-norm if it satisfies the following conditions:

$\left(T_{1}\right) \mathcal{T}(1, a)=a$ and $\mathcal{T}(0, a)=0$ for all $a \in L$;

$\left(T_{2}\right) b \leqslant c \Rightarrow \mathcal{T}(a, b) \leqslant \mathcal{T}(a, c)$ for all $a, b, c \in L$.

A pseudo-t-norm $\mathcal{T}$ on $L$ is said to be infinitely $\checkmark$-distributive if it satisfies the following condition: $a, b_{j} \in L(j \in \Gamma) \Rightarrow \mathcal{T}\left(a, \vee_{j \in \Gamma} b_{j}\right)=\vee_{j \in \Gamma} \mathcal{T}\left(a, b_{j}\right)$.

A pseudo-t-norm $\mathcal{T}$ on $L$ is said to be infinitely $\wedge$-distributive if it satisfies the following condition: $a, b_{j} \in L(j \in \Gamma) \Rightarrow \mathcal{T}\left(a, \wedge_{j \in \Gamma} b_{j}\right)=\wedge_{j \in \Gamma} \mathcal{T}\left(a, b_{j}\right)$.

A pseudo-t-norm $\mathcal{T}$ on $L$ is said to be infinitely distributive if it is both infinitely $\vee$-distributive and $\wedge$-distributive.

(2) A binary operation $\mathcal{I}$ on $L$ is called to be an implication if it satisfies the following conditions:

$\left(I_{1}\right) \mathcal{I}(0,0)=\mathcal{I}(1,1)=1$ and $\mathcal{I}(1,0)=0$;

$\left(I_{2}\right) b \leqslant c \Rightarrow \mathcal{I}(a, b) \leqslant \mathcal{I}(a, c)$ and $\mathcal{I}(b, a) \geqslant \mathcal{I}(c, a)$ for all $a, b, c \in L$.

An implication $\mathcal{I}$ on $L$ is said to satisfy the left neutrality property if $\mathcal{I}(1, b)=b$ for every $b \in[0,1]$; be infinitely $\vee$-distributive if it satisfies the following condition:

$a, b_{j} \in L(j \in \Gamma) \Rightarrow \mathcal{I}\left(a, \vee_{j \in \Gamma} b_{j}\right)=\vee_{j \in \Gamma} \mathcal{I}\left(a, b_{j}\right)$.

An implication $\mathcal{I}$ on $L$ is said to be infinitely $\wedge$ distributive if it satisfies the following condition: $a, b_{j} \in L(j \in \Gamma) \Rightarrow \mathcal{I}\left(a, \wedge_{j \in \Gamma} b_{j}\right)=\wedge_{j \in \Gamma} \mathcal{I}\left(a, b_{j}\right)$.
An implication $\mathcal{I}$ on $L$ is called infinitely distributive if it is both infinitely $\vee$-distributive and $\wedge$ distributive.

Remark 2.1 Note that for any implication $\mathcal{I}$, it holds that $\mathcal{I}(a, 1)=\mathcal{I}(0, b)=1$ for every $a, b \in$ $[0,1]$.

Definition 2.2 [27] Let $\varphi$ be a mapping from $L \times L$ into L. Define $\mathcal{I}_{\varphi}, \mathcal{L}_{\varphi}$ as follows:

$\mathcal{I}_{\varphi}(a, b)=\sup \{x \in L: \varphi(a, x) \leqslant b\}$ for all $a, b \in L$,

$\mathcal{L}_{\varphi}(a, b)=\inf \{x \in L: \varphi(a, x) \geqslant b\}$ for all $a, b \in L$.

It is tacitly assumed that $\vee \emptyset=0$ and $\wedge \emptyset=1$.

Remark 2.2 In Definition 2.2, when $\varphi$ is a conjunctor $\mathcal{T}$ or an implication $\mathcal{I}$, we can obtain operators $\mathcal{I}_{\mathcal{T}}$ or $\mathcal{I}_{\mathcal{I}}$. Further, $\mathcal{I}_{\mathcal{T}}$ is an implication.

Lemma 2.1 [10] If $\mathcal{T}$ is an infinitely $\vee$ distributive conjunctor on $L$, then the following conditions are equivalent:

(1) $\mathcal{T}(a, c) \leqslant b \Leftrightarrow c \leqslant \mathcal{I}_{\mathcal{T}}(a, b)$ for all $a, b \in L$.

(2) $\mathcal{T}(a, 0)=0$ for all $a \in L$.

Example 2.1 From five pseudo-t-norms in Table 1, we can obtain the following five implications (see Table 2). In fact, $\mathcal{T}_{Y}(a, b) \neq \mathcal{T}_{Y}(b, a)$ when $a=0.3$, $b=0.8$, hence $\mathcal{T}_{Y}$ is not a t-norm. Further, these implications satisfy the left neutrality property.

From now on, unless otherwise stated, we always assume that $\mathcal{T}$ is an infinitely $\vee$-distributive pseudot-norm and $\mathcal{I}$ is an infinitely $\wedge$-distributive implication with $\mathcal{T}(a, 0)=\mathcal{T}(0, a)=0$ and $\mathcal{I}(a, 1)=1$, $\forall a \in L$.

Lemma 2.2 [28] (1) If $\mathscr{X}_{1} \triangleq\{x \in L: \mathcal{I}(a, x)=$ $b\} \neq \emptyset$, then the equation $\mathcal{I}(a, x)=b$ has the smallest solution $\wedge \mathscr{X}_{1}$ and $\wedge \mathscr{X}_{1}=\mathcal{T}(a, b)$.

(2) If $\mathcal{I}_{\mathcal{T}}$ is $\vee$-distributive, i.e. $\mathcal{I}\left(x, x_{1} \vee x_{2}\right)=$ $\mathcal{I}\left(x, x_{1}\right) \vee \mathcal{I}\left(x, x_{2}\right)$ for all $x, x_{1}, x_{2} \in L$, then $\mathscr{X}_{1}$ is a convex sublattice of $L$ when $\mathscr{X}_{1} \neq \emptyset$. Moreover, if $\mathcal{I}$ $i s$ an infinitely $\vee$-distributive implication and $\mathscr{X}_{1} \neq$ $\emptyset$, then the equation $\mathcal{I}(a, x)=b$ has the greatest solution $\vee \mathscr{X}_{1}$ and $\vee \mathscr{X}_{1}=\mathcal{I}_{\mathcal{I}_{\mathcal{T}}}(a, b)$.

Denote

$$
X_{*}=\left(x_{j *}\right)_{j \in J} \triangleq\left(\max _{i \in I} \mathcal{T}\left(a_{i j}, b_{i}\right)\right)_{j \in J} .
$$

Lemma 2.3 [30] System (2) is solvable if and only if $X_{*}$ is a solution of system (2), and $X_{*}$ is the smallest solution of system (2).

\section{Characteristic matrices of system (2) and attainable components}

In this section, we give the concepts of characteristic matrices and attainable components. Further, some properties are described. 


\begin{tabular}{|c|c|c|}
\hline Name & Year & Formula \\
\hline Minimum & $1932[1,13]$ & $\mathcal{T}_{M}(a, b)=\min (a, b)$ \\
$\begin{array}{c}\mathcal{T}_{P}(a, b)=a b \\
\text { Algebraic product }\end{array}$ & $1969[9,13]$ & $\mathcal{T}_{L}(a, b)=\max \{0, a+b-1\}$ \\
Eukasiewicz t-norm & $1923[1,13]$ & $\mathcal{T}_{Y}(a, b)= \begin{cases}b^{\frac{1}{a}}, & \text { if } a \cdot b>0, \\
0, & \text { if } a \cdot b=0 .\end{cases}$ \\
Yager operation & $1980[31]$ & $\begin{array}{ll}0, & \text { if } a+b \leqslant 1, \\
a \wedge b, & \text { otherwise. }\end{array}$ \\
\hline
\end{tabular}

Table 1: lists a few of common pseudo-t-norms.

\begin{tabular}{|c|c|c|}
\hline Name & Year & Formula \\
\hline Gödel & $1932[1]$ & $\mathcal{I}_{\mathcal{T}_{M}}(a, b)= \begin{cases}1, & \text { if } a \leqslant b, \\
b, & \text { otherwise. }\end{cases}$ \\
Goguen & $1969[9]$ & $\mathcal{I}_{\mathcal{T}_{P}}(a, b)= \begin{cases}1, & \text { if } a \leqslant b, \\
\frac{b}{a}, & \text { otherwise. }\end{cases}$ \\
Eukasiewicz & $1923[1]$ & $\mathcal{I}_{\mathcal{T}_{L}}(a, b)=\min \{1,1-a+b\}$. \\
Yager & $1980[31]$ & $\mathcal{I}_{\mathcal{T}_{Y}}(a, b)= \begin{cases}b^{a}, & a>0 \text { or } b>0, \\
1, & a=0 \text { and } b=0 .\end{cases}$ \\
Fodor & $1993[8]$ & $\mathcal{I}_{\mathcal{T}_{F}}(a, b)= \begin{cases}1, & \text { if } a \leqslant b, \\
(1-a) \vee b, & \text { otherwise. }\end{cases}$ \\
\hline
\end{tabular}

Table 2: examples of implications corresponding to the Table 1.

The complete solution set $\mathscr{X}$, when it is not empty, can be well characterized and determined by a unique smallest solution and a finite number of maximal ones [30], i.e.

$$
\mathscr{X}=\cup_{X^{0} \in \mathscr{X}^{0}}\left[X_{*}, X^{0}\right],
$$

where $\mathscr{X}^{0}$ denotes the set of all maximal solutions of system (2).

Lemma 3.1 If $b_{i}=1$ for all $i \in I$, then $\mathscr{X}^{0}$ is $\left\{(1,1, \cdots, 1)^{\prime}\right\}$.

In the following, we may assume that there exists $i_{0} \in I$ such that $b_{i_{0}}<1$.

Example 3.1 Consider the following fuzzy relational equations with inf- $\mathcal{I}_{\mathcal{T}_{L}}$ composition $A \circ_{\mathcal{I}_{\mathcal{T}_{L}}}$ $X=B$, where

$$
A=\left(\begin{array}{llllll}
0.5 & 0.7 & 0.2 & 0.3 & 0.7 & 0.8 \\
0.9 & 0.4 & 0.6 & 0.5 & 0.4 & 0.7 \\
0.4 & 0.6 & 0.3 & 0.3 & 0.1 & 0.8 \\
0.1 & 0.8 & 0.4 & 0.1 & 1.0 & 0.2 \\
0.6 & 0.2 & 0.3 & 0.2 & 0.4 & 0.5
\end{array}\right) \text {, }
$$

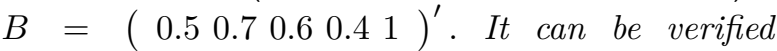
that the system is solvable and its solution is $X_{*}=(0.6,0.2,0.3,0.2,0.4,0.5)^{\prime}$.

Lemma 3.2 If $\mathscr{X} \neq \emptyset$, then for each $i \in I$, there exists at least one index $j_{i} \in J$ such that $\mathcal{I}\left(a_{i j_{i}}, x_{j_{i}}\right)=b_{i}$ for any $X=\left(x_{j}\right)_{j \in J} \in \mathscr{X}$.

Proof. It is trivial since $J$ is a finite index set.

Definition 3.1 For the smallest solution $X_{*}=$ $\left(x_{j *}\right)_{j \in J}$, the characteristic matrix $\widetilde{Q}=\left(\widetilde{q}_{i j}\right)_{m \times n}$ of the system (2) is defined as

$$
\widetilde{q}_{i j}= \begin{cases}{\left[x_{j *}, \mathcal{I}_{\mathcal{I}}\left(a_{i j}, b_{i}\right)\right],} & \text { if } \mathcal{I}\left(a_{i j}, x_{j *}\right)=b_{i}, \\ \emptyset, & \text { otherwise. }\end{cases}
$$

for all $i \in I$ and $j \in J$.

By Lemma 2.2, each element $\widetilde{q}_{i j}$ of the characteristic matrix $\widetilde{Q}$ indicates all the possible values for the component $x_{j}$ to satisfy the $i$-th equation of system (2). The system (2) is solvable if and only if each row of $\widetilde{Q}$ is a nonempty row, i.e. each row contains at least one nonempty element.

Example 3.2 (Continue from Example 3.1) The characteristic matrix $\widetilde{Q}$ is

$\widetilde{Q}=\left(\begin{array}{cccccc}\emptyset & 0.2 & \emptyset & \emptyset & \emptyset & \emptyset \\ 0.6 & \emptyset & 0.3 & 0.2 & \emptyset & \emptyset \\ \emptyset & 0.2 & \emptyset & \emptyset & \emptyset & \emptyset \\ \emptyset & 0.2 & \emptyset & \emptyset & 0.4 & \emptyset \\ {[0.6,1]} & {[0.2,1]} & {[0.3,1]} & {[0.2,1]} & {[0.4,1]} & {[0.5,1]}\end{array}\right)$.

Lemma 3.3 Let $X=\left(x_{j}\right)_{j \in J} \in \mathscr{X}$. If the component $x_{j}$ satisfies $\mathcal{I}\left(a_{k j}, x_{j}\right)=b_{k}$ for some $k \in I$, then the corresponding component $x_{j *}$ in smallest solution also satisfies $\mathcal{I}\left(a_{k j}, x_{j *}\right)=b_{k}$.

Proof. By Definition 2.1, $\quad \mathcal{I}\left(a_{k j}, x_{j *}\right) \leqslant$ $\mathcal{I}\left(a_{k j}, x_{j}\right)=b_{k}$ since $X_{*} \leqslant X . \quad$ Вy $\inf _{j \in J} \mathcal{I}\left(a_{i j}, x_{j *}\right)=b_{i}$ for all $i \in I$, we have $\mathcal{I}\left(a_{i j}, x_{j *}\right) \geqslant b_{i}$ for all $i \in I$. Therefore, $\mathcal{I}\left(a_{k j}, x_{j *}\right)=b_{k}$.

Definition 3.2 Let $X=\left(x_{j}\right)_{j \in J} \in L^{n}$. The component $x_{j_{0}}$ of $X$ is called attainable if $\mathcal{I}\left(a_{i j_{0}}, x_{j_{0}}\right)=$ $b_{i}$ holds for some $i \in I$; otherwise, $x_{j_{0}}$ is called unattainable.

The equations of a system can be satisfied only by attainable components. The presence of attainable components is a necessary condition that the solution set is nonempty. If a system has no attainable 
components, then its solution set is empty. But if a system has attainable component, then the solution set is not sure. The values of all unattainable components in all maximal solutions are obviously equal to 1. However, some attainable components also take only 1 in maximal solutions. The column of the characteristic matrix corresponding to an attainable component contains at leat one nonempty element. For the smallest solution, the following result holds.

Lemma 3.4 If $\mathscr{X} \neq \emptyset$, then every component of the smallest solution is attainable.

Proof. It is straightforward from expression (3) and Definition 3.2.

Theorem 3.5 Let $X=\left(x_{j}\right)_{j \in J} \in L^{n}$. Then $X$ is a solution of system (2) if and only if $X \geqslant X_{*}$ and the induced binary matrix $Q_{X}=\left(q_{i j}^{\prime}\right)_{m \times n}$ has no zero rows, where

$$
q_{i j}^{\prime}= \begin{cases}1, & \text { if } x_{j} \in \widetilde{q}_{i j} \\ 0, & \text { otherwise }\end{cases}
$$

Proof. If $X$ is a solution of system (2), then $X \geqslant$ $X_{*}$ by Lemma 2.3. Further, there exists an index $j_{i} \in J$ such that $\mathcal{I}\left(a_{i j_{i}}, x_{j_{i}}\right)=b_{i}$ for each $i \in I$. Therefore, $x_{j_{i}} \in \widetilde{q}_{i j_{i}}$. Thus $q_{i j_{i}}^{\prime}=1$ for each $i \in I$, i.e. $Q_{X}$ has no zero rows.

The converse implication, if $X_{*} \leqslant X$ and $Q_{X}$ has no zero rows, then

$$
b_{i} \leqslant \mathcal{I}\left(a_{i j}, x_{j *}\right) \leqslant \mathcal{I}\left(a_{i j}, x_{j}\right) \text { for all } i \in I .
$$

Further, by the definition of $Q_{X}$, for every $i \in I$, there exists an index $j_{i} \in J$ such that $q_{i j_{i}}^{\prime}=1$, that is to say $x_{j_{i}} \leqslant \mathcal{I}_{\mathcal{I}}\left(a_{i j_{i}}, b_{i}\right)$. Therefore, for every $i \in I$, we have

$$
\begin{aligned}
\inf _{j \in J} \mathcal{I}\left(a_{i j}, x_{j}\right) & =\left[\inf _{j \in J \backslash\left\{j_{i}\right\}} \mathcal{I}\left(a_{i j}, x_{j}\right)\right] \wedge\left[\mathcal{I}\left(a_{i j_{i}}, \mathcal{I}_{\mathcal{I}}\left(a_{i j_{i}}, b_{i}\right)\right)\right. \\
& \leqslant \mathcal{I}\left(a_{i j_{i}}, \mathcal{I}_{\mathcal{I}}\left(a_{i j_{i}}, b_{i}\right)\right) \\
& =\mathcal{I}\left(a_{i j_{i}}, \sup \left\{x \in L: \mathcal{I}\left(a_{i j_{i}}, x\right) \leqslant b_{i}\right\}\right) \\
& =\sup \left\{\mathcal{I}\left(a_{i j_{i}}, x\right): \mathcal{I}\left(a_{i j_{i}}, x\right) \leqslant b_{i}\right\} \\
& \leqslant b_{i} .
\end{aligned}
$$

Therefore, by formulas (6) and (7), $\inf _{j \in J} \mathcal{I}\left(a_{i j}, x_{j}\right)=b_{i}$ for all $i \in I$, i.e. $X \in \mathscr{X}$ holds.

\section{Definition 3.3 Let $a, b, c \in L$.}

(i) The implication $\mathcal{I}$ is said to satisfy the right cancelation law, if

$$
\mathcal{I}(a, b)=\mathcal{I}(a, c) \text { implies } a=0 \text { or } b=c .
$$

(ii) The implication $\mathcal{I}$ is said to satisfy the right conditional cancelation law, if

$$
\mathcal{I}(a, b)=\mathcal{I}(a, c)<1 \text { implies } b=c .
$$

If an implication $\mathcal{I}$ satisfies the right cancelation law, it obviously fulfills the right conditional cancelation law, but the inverse is not true. In fact, the right cancelation law and the right conditional cancelation law of implications require that the implication be strictly increasing in their second arguments with proper domains. It is easy to see that the first four implications in Example 2.1, i.e. the Gödel implication $\mathcal{I}_{\mathcal{T}_{M}}$, the Goguen implication $\mathcal{I}_{\mathcal{T}_{P}}$, the Eukasiewicz implication $\mathcal{I}_{\mathcal{T}_{L}}$ and the Yager implication $\mathcal{I}_{\mathcal{T}_{Y}}$ satisfy the right conditional cancelation law. However, the Fodor implication $\mathcal{I}_{\mathcal{T}_{F}}$ do not satisfy the right conditional cancelation law since $\mathcal{I}_{\mathcal{T}_{F}}(0.6,0.2)=\mathcal{I}_{\mathcal{T}_{F}}(0.6,0.3)=$ 0.4 but $0.2 \neq 0.3$. Next, we have the following result.

Lemma 3.6 Let $\mathcal{I}$ satisfy the right conditional cancelation law and its second partial mapping $\mathcal{I}(a, \cdot)$ be infinitely distributive. Then the equation $\mathcal{I}(a, x)=b$ with $b \in[0,1)$ has a unique solution $\mathcal{I}_{\mathcal{I}}(a, b)$ and $\mathcal{I}_{\mathcal{I}}(a, b) \neq 1$.

Lemma 3.7 Let $\mathcal{I}$ satisfy the right conditional cancelation law, $0 \leqslant b_{i}<1$ for all $i \in I$ and $X=$ $\left(x_{j}\right)_{j \in J} \in \mathscr{X}$. If the component $x_{j}$ is attainable, then $x_{j}=x_{j *}<1$.

Proof. Since $x_{j}$ is attainable, therefore $\mathcal{I}\left(a_{i j}, x_{j}\right)=$ $b_{i}$ holds for some $i \in I$. By Lemma 3.3, we have $\mathcal{I}\left(a_{i j}, x_{j}\right)=\mathcal{I}\left(a_{i j}, x_{j *}\right)=b_{i}$. Since $b_{i}<1$ for all $i \in$ $I$ and $\mathcal{I}$ satisfies the right conditional cancelation law, therefore $\mathcal{I}\left(a_{i j}, x_{j}\right)=b_{i}$ has a unique solution $x_{j} \in[0,1)$ by Lemma 3.6. Thus $x_{j}=x_{j *}<1$.

Lemma 3.8 Let $X=\left(x_{j}\right)_{j \in J} \in \mathscr{X}^{0}$. If $x_{j}<1$ for some $j \in J$, then $x_{j}=x_{j *}$.

Proof. If $x_{j}$ is attainable in the $i$-th equation with $b_{i}<1$, then $x_{j}=x_{j *}<1$ by Lemma 3.7. On the other hand, if $x_{j}$ is only attainable in the equation )] with $b_{i}=1$, then $\mathcal{I}\left(a_{i j}, x_{j}\right)>b_{i}$ for all $b_{i}<1$. Hence, $\mathcal{I}\left(a_{i j}, 1\right) \geqslant \mathcal{I}\left(a_{i j}, x_{j}\right)>b_{i}$ for all $b_{i}<1$. Therefore, $x_{j}=1$ since $X \in \mathscr{X}^{0}$.

Lemma 3.9 Let $0 \leqslant b_{i}<1$ for all $i \in I$ and $X^{0}=$ $\left(x_{j}^{0}\right)_{j \in J} \in \mathscr{X}^{0}$. Then $x_{j^{\prime}}^{0}$ is unattainable if and only if $x_{j^{\prime}}^{0}=1$.

Proof. If $x_{j^{\prime}}^{0}=1$, then $\mathcal{I}\left(a_{i j}, 1\right)=1>b_{i}$ for all $i \in I$ by Remark 2.1. Therefore, $x_{j^{\prime}}^{0}$ is unattainable.

Conversely, we assume that $x_{j^{\prime}}^{0}$ is unattainable and $x_{j^{\prime}}^{0}<1$ for some $j^{\prime} \in J$. For each $X^{0}=$ $\left(x_{j}^{0}\right)_{j \in J} \in \mathscr{X}^{0}$, we have $\inf _{j \in J \backslash\left\{j^{\prime}\right\}} \mathcal{I}\left(a_{i j}, x_{j}^{0}\right)=$ $b_{i}$ for all $i \in I$. Define $X=\left(x_{j}\right)_{j \in J}$ with

$$
x_{j}= \begin{cases}1, & \text { if } j=j^{\prime} \\ x_{j}^{0}, & \text { otherwise. }\end{cases}
$$

Therefore, we have $\inf _{j \in J} \mathcal{I}\left(a_{i j}, x_{j}\right)=$ $\left[\inf _{j \in J \backslash\left\{j^{\prime}\right\}} \mathcal{I}\left(a_{i j}, x_{j}^{0}\right)\right] \wedge \mathcal{I}\left(a_{i j^{\prime}}, 1\right)=b_{i}$ for all $i \in I$, i.e. $X \in \mathscr{X}$. Further $X \geqslant X^{0}$ and $X \neq X^{0}$, which contradicts with that $X^{0} \in \mathscr{X}^{0}$.

From Lemmas 3.7, 3.8 and 3.9, the following result holds. 
Theorem 3.10 Let $\mathcal{I}$ satisfy the right conditional cancelation law and $0 \leqslant b_{i}<1$ for all $i \in I$. For any $X^{0}=\left(x_{j}^{0}\right)_{j \in J} \in \mathscr{X}^{0}$, if $x_{j}^{0}$ is attainable, then $x_{j}^{0}=x_{j *}<1$; otherwise, $x_{j}^{0}=1$.

Next, we consider the system (2) without the restriction $b_{i}<1$ for all $i \in I$. Let $I\left(x_{j}\right) \triangleq\{i \in$ $\left.I: \mathcal{I}\left(a_{i j}, x_{j}\right)=b_{i}\right\}$.

Definition 3.4 Let $X=\left(x_{j}\right)_{j \in J} \in \mathscr{X} . A n$ attainable component $x_{j}$ is called 1-type if $b_{i}=1$ for each $i \in I\left(x_{j}\right)$; otherwise, $x_{j}$ is called 2-type.

Lemma 3.11 Let $X=\left(x_{j}\right)_{j \in J} \in \mathscr{X}$. If $b_{k}=1$ for some $k \in I$, then $\mathcal{I}\left(a_{k j}, x_{j}\right)=1$ holds for all $j \in J$.

Proof. Since $b_{k}=1$ for some $k \in I$, then

$$
\inf \left\{\mathcal{I}\left(a_{k 1}, x_{1}\right), \mathcal{I}\left(a_{k 2}, x_{2}\right), \cdots, \mathcal{I}\left(a_{k n}, x_{n}\right)\right\}=1 .
$$

Therefore, $\mathcal{I}\left(a_{k j}, x_{j}\right)=1$ holds for all $j \in J$.

Lemma 3.12 Let $\mathcal{I}$ satisfy the right conditional cancelation law. For any $X=\left(x_{j}\right)_{j \in J} \in \mathscr{X}$, if a component $x_{j}$ is a 2-type attainable, then $x_{j}=$ $x_{j *}<1$.

Proof. The proof is similar to that of Lemma 3.7.

Lemma 3.13 For each $X^{0}=\left(x_{j}^{0}\right)_{j \in J} \in \mathscr{X}^{0}, a$ component $x_{j}^{0}=1$ if and only if $x_{j}^{0}$ is either 1-type attainable or an unattainable.

Proof. If $x_{j}^{0}=1$, then $\mathcal{I}\left(a_{i j}, x_{j}^{0}\right)=\mathcal{I}\left(a_{i j}, 1\right)=1$ for all $i \in I$ by Remark 2.1. Therefore, if $b_{i}=1$ for some $i \in I$, then $x_{j}^{0}$ is 1-type attainable; if $b_{i}<1$ for all $i \in I$, then $x_{j}^{0}$ is unattainable.

Conversely, if $x_{j}^{0}$ is 1-type attainable, then $\mathcal{I}\left(a_{i j}, x_{j}^{0}\right)=1$. Therefore, $x_{j}^{0}=1$ since $\mathcal{I}\left(a_{i j}, 1\right)=1$ and $X^{0}=\left(x_{j}^{0}\right)_{j \in J} \in \mathscr{X}^{0}$. On the other hand, if $x_{j}^{0}$ is unattainable, we assume that $x_{j}^{0}<1$, then the rest of the proof is similar to that of Lemma 3.9.

From Lemmas 3.12 and 3.13, the following theorem is true.

Theorem 3.14 Let $\mathcal{I}$ satisfy the right conditional cancelation law. For any $X^{0}=\left(x_{j}^{0}\right)_{j \in J} \in \mathscr{X}^{0}$, if a component $x_{j}^{0}$ is 2-type attainable, then $x_{j}^{0}=x_{j *}<$ 1 ; otherwise, $x_{j}^{0}=1$.

\section{Relation between maximal solutions and irredundant coverings}

In this section, we discuss the problem of finding all maximal solutions of system (2). Since system (2) has at most one smallest solution, by Theorem 3.14, fuzzy relational equations with the right conditional cancelation law implication have a finite number of maximal solutions. To describe the set of all solutions, we have to describe the set of all maximal solutions. Next, we transform the problem of finding all maximal solutions of system (2) into that of finding all irredundant coverings of the characteristic matrix of system (2).
Definition 4.1 [14] Let $\widetilde{Q}$ be a characteristic matrix of system (2).

i) A column $j$ is said to cover a row $i$ if $\widetilde{q}_{i j} \neq \emptyset$. $\underset{\sim}{A}$ set of nonempty columns $C$ forms a covering of $\widetilde{Q}$ if each row of $\widetilde{Q}$ is covered by some column in $C$.

ii) A column $j$ in a covering $C$ is called redundant if the set of columns $C \backslash\{j\}$ remains to be a covering of $\widetilde{Q}$. A covering $C$ is irredundant if it has no redundant columns.

The set of all irredundant coverings of a characteristic matrix $\widetilde{Q}$ is denoted by $\Phi(\widetilde{Q})$. Therefore, in Example 3.2 (ii) the set of all irredundant coverings of $\widetilde{Q}$ is $\Phi(\widetilde{Q})=\{\{1,2\},\{1,3\}\}$. By Theorem 3.5, we have

Theorem 4.1 System (2) is solvable if and only if there exists a covering $C$ of the characteristic matrix $\widetilde{Q}$.

Definition 4.2 Let $C \in \Phi(\widetilde{Q})$. The mapping vector of $C$ is denoted by $X^{C}=\left(x_{j}^{c}\right)_{j \in J}$ with

$$
x_{j}^{c}= \begin{cases}1, & \text { if } j \notin C, \\ x_{j *}, & \text { otherwise } .\end{cases}
$$

Definition 4.3 Let $X=\left(x_{j}\right)_{j \in J} \in \mathscr{X}$. The attainable index set of $X$ is denoted by $\Gamma^{X} \triangleq\{j \in$ $\left.J: x_{j}=x_{j *}<1\right\}$.

Example 4.1 (Continue from Examples 3.1 and 3.2 (i)) For the covering $C=\{1,2\}$, the mapping vector of $C$ is $X^{C}=(0.6,0.2,1,1,1,1)^{\prime}$. Let $X=$ $(1,0.2,0.8,1,0.6,0.8)^{\prime} \in \mathscr{X}$, the attainable index set is $\Gamma^{X}=\{1,4\}$.

Denote $I_{j}(\widetilde{Q})=\left\{i \in I: \widetilde{q}_{i j} \neq \emptyset\right\}$ for all $j \in J$ and $J_{i}(\widetilde{Q})=\left\{j \in J: \widetilde{q}_{i j} \neq \emptyset\right\}$ for all $i \in I$.

Lemma 4.2 Let $\mathscr{X} \neq \emptyset$ and $C \in \Phi(\widetilde{Q})$. Then $x_{j *} \neq 1$ holds for all $j \in C$, where $X_{*}=\left(x_{j *}\right)_{j \in J}$ is the smallest solution of systems (2).

Proof. Assume that there exists $j_{0} \in C$ such that $x_{j_{0} *}=1$, then by Lemma 3.3 for each $i \in I_{j_{0}}(\widetilde{Q})$, we have $\widetilde{q}_{i j_{0}} \neq \emptyset$ since $\mathscr{X} \neq \emptyset$. Further, $\mathcal{I}\left(a_{i j_{0}}, x_{j_{0} *}\right)=$ $\mathcal{I}\left(a_{i j_{0}}, 1\right)=1$. Therefore, $b_{i}=1$ for all $i \in I_{j_{0}}(\widetilde{Q})$. Since $\mathscr{X} \neq \emptyset$, we have $\widetilde{q}_{i j} \neq \emptyset$ for all $i \in I_{j_{0}}(\widetilde{Q})$ and $j \in J$. Hence $I_{j_{0}}(\widetilde{Q}) \subseteq I_{j}(\widetilde{Q})$ for all $j \in J$. Since $C$ is an irredundant covering of $\widetilde{Q}$, therefore we must have $\widetilde{q}_{i_{0} j_{i_{0}}} \neq \emptyset$ for some $j_{i_{0}}$ when $b_{i_{0}}<1$ for some $i_{0} \in I$. Note that $\mathcal{I}\left(a_{i_{0} j_{0}}, x_{j_{0} *}\right)=\mathcal{I}\left(a_{i_{0} j_{0}}, 1\right)=$ $1>b_{i_{0}}$, then $\widetilde{q}_{i_{0} j_{0}}=\emptyset$. Further, $j_{i_{0}} \neq j_{0}$. Thus $C \backslash\left\{j_{0}\right\} \neq \emptyset$. Therefore, $\cup_{j \in C \backslash\left\{j_{0}\right\}} I_{j}(\widetilde{Q})=I$ since $C \backslash\left\{j_{0}\right\} \neq \emptyset$ and $I_{j_{0}}(\widetilde{Q}) \subseteq I_{j}(\widetilde{Q})$, which contradicts $C \in \Phi(\widetilde{Q})$.

Lemma 4.3 Let $\mathcal{I}$ satisfy the right conditional cancelation law and $X=\left(x_{j}\right)_{j \in J} \in \mathscr{X}$. Then $\Gamma^{X}$ is a covering of $\widetilde{Q}$. 
Proof. Let $I^{\prime}=\left\{i \in I: b_{i} \neq 1\right\}$. It is obvious that $I^{\prime} \neq \emptyset$. By Lemma 3.12, then $\Gamma^{X} \neq \emptyset$. Since $X=\left(x_{j}\right)_{j \in J} \in \mathscr{X}$, there exists an index $j \in J$ such that $\mathcal{I}\left(a_{i j}, x_{j}\right)=b_{i}$ for each $i \in I^{\prime}$. For any $j \in \Gamma^{X}$, we have $\mathcal{I}\left(a_{i j}, x_{j}\right)=\mathcal{I}\left(a_{i j}, x_{j *}\right)=b_{i}<1$ by Lemma 3.12. Thus $\widetilde{q}_{i j} \neq \emptyset$ by Definition 3.1. Consequently, $i \in I_{j}(\widetilde{Q})$. Note that, $b_{i_{0}}=1$ for all $i_{0} \in I \backslash I^{\prime} \neq \emptyset$ and $X=\left(x_{j}\right)_{j \in J} \in \mathscr{X}$, then $\widetilde{q}_{i_{0} j} \neq \emptyset$ for all $j \in J$. Further, $\widetilde{q}_{i_{0} j} \neq \emptyset$ for all $j \in \Gamma^{X} \subseteq J$. Thus, $\cup_{j \in \Gamma^{X}} I_{j}(\widetilde{Q})=I^{\prime} \cup\left(I \backslash I^{\prime}\right)=I$. That is to say, $\Gamma^{X}$ is a covering of $\widetilde{Q}$.

Theorem 4.4 If $\mathscr{X} \neq \emptyset$ and $b_{i}<1$ for all $i \in$ $I$, then there exists a one-to-one mapping between maximal solutions of system (2) and irredundant coverings.

Proof. We prove it from the following three procedures.

First, we prove that if $C$ is an irredundant covering, then $X^{C} \in \mathscr{X}^{0}$. Let $C$ be an irredundant covering. Then $\cup_{j \in C} I\left(x_{j *}\right)=I$. Further, there exists $j \in J$ such that $\mathcal{I}\left(a_{i j}, x_{j *}\right)=b_{i}$ for each $i \in I$. By formula (10), we have that $\mathcal{I}\left(a_{i j}, x_{j}^{c}\right)=b_{i}$ for each $i \in I$. That is to say $X^{C} \in \mathscr{X}$. Next, we show that $X^{C}$ is a maximal solution of system (2). Assume to the contrary that $X^{C} \notin \mathscr{X}^{0}$. There exists $k \in J$ such that $\mathcal{I}\left(a_{i k_{i}}, x_{k_{i}}\right)>b_{i}$ for every $i \in I$. By Lemma 3.8, we can define $X(k)=\left(x_{j}\right)_{j \in J}$ with

$$
x_{j}= \begin{cases}1, & \text { if } j=k, \\ x_{j}^{c}, & \text { otherwise }\end{cases}
$$

Therefore, we have $C \backslash\{k\}$ is a covering, which contradicts with that $C$ is an irredundant covering. Hence $X^{C} \in \mathscr{X}^{0}$.

Second, we prove that if $X=\left(x_{j}\right)_{j \in J} \in \mathscr{X}^{0}$, then $J_{c} \triangleq\left\{j \in J: x_{j}<1\right\}$ is an irredundant covering. By $X=\left(x_{j}\right)_{j \in J} \in \mathscr{X}^{0}$, then $\inf _{j \in J} \mathcal{I}\left(a_{i j}, x_{j}\right)=b_{i}$ for all $i \in I$. Hence, for each $i \in I$, there exists $j \in J_{c}$ such that $\mathcal{I}\left(a_{i j}, x_{j}\right)=b_{i}$. By Lemma 3.8, we have $\mathcal{I}\left(a_{i j}, x_{j *}\right)=b_{i}$. Therefore, $\cup_{j \in J_{c}} I\left(x_{j *}\right)=$ I. By Definition 4.1, we have $J_{c}$ is a covering. If $\cup_{j \in J_{c} \backslash\left\{j_{0}\right\}} I\left(x_{j *}\right)=I$ for some $j_{0} \in J$, then for each $i \in I$, there exists $j \in J_{c} \backslash\left\{j_{0}\right\}$ such that $\mathcal{I}\left(a_{i j}, x_{j}\right)=$ $\mathcal{I}\left(a_{i j}, x_{j *}\right)=b_{i}$. Define $X^{\prime}\left(j_{0}\right)=\left(x_{j}^{\prime}\right)_{j \in J}$ with

$$
x_{j}^{\prime}= \begin{cases}1, & \text { if } j=j_{0}, \\ x_{j}, & \text { otherwise }\end{cases}
$$

Obviously, $X^{\prime}\left(j_{0}\right) \in \mathscr{X}$ and $X^{\prime}\left(j_{0}\right) \geqslant X$ and $X^{\prime}\left(j_{0}\right) \neq X$, which contradicts with that $X \in \mathscr{X}^{0}$. Therefore, $J_{c}$ is an irredundant covering.

At last, we can construct a one-to-one mapping $f: C \rightarrow X^{C}$ with $x_{j}^{c}=x_{j *}$ for $j \in C$ and $x_{j *}=1$ for $j \notin C$. Hence, there is a one-to-one mapping between maximal solutions of system (2) and irredundant coverings.

From Theorem 4.4, we have the following corollaries.
Corollary 4.5 For any $C^{1}, C^{2} \in \Phi(\widetilde{Q})$, if $C^{1} \neq$ $C^{2}$, then $X^{C^{1}} \neq X^{C^{2}}$.

Corollary 4.6 For any $X^{1}, X^{2} \in \mathscr{X}^{0}$, if $X^{1} \neq$ $X^{2}$, then $\Gamma^{X^{1}} \neq \Gamma^{X^{2}}$.

Corollary 4.7 All maximal solutions of system (2) have the following form $X^{0}=\left(x_{j}^{0}\right)_{j \in J}$ with

$$
x_{j}^{0}= \begin{cases}x_{j *}, & \text { if } j \in C, \\ 1, & \text { otherwise, }\end{cases}
$$

where $C \in \Phi(\widetilde{Q})$.

Thus by Lemma 2.3, Theorem 4.4 and Corollaries 4.5, 4.6 and 4.7, we can obtain the solution set of system (2). In fact, maximal solutions of system (2) are completely determined by irredundant coverings of the characteristic matrix. Shieh [26] proposed an algorithm for finding irredundant covering of a matrix. Base the algorithm, next we give an example for finding all maximal solutions. The specific algorithm may see [26].

Example 4.2 (Continue from Example 3.2) By the algorithm in [26], we have $\Phi(\widetilde{Q})=\{\{2,1\},\{2,3\},\{2,4\}\} . \quad$ Consequently, all maximal solutions are $\mathscr{X}^{*}=$ $\left\{(0.6,0.2,1,1,1,1)^{\prime},(1,0.2,0.3,1,1,1)^{\prime},(1,0.2,1,0.2,1,1)^{\prime}\right\}$.

\section{The uniqueness of solution of system (2)}

In this section, we give two necessary and sufficient conditions that the system (2) has a unique(maximal) solution.

Definition 5.1 Let $\widetilde{Q}$ be a characteristic matrix of system (2). A column $j$ of $\widetilde{Q}$ is said to be in the kernel $\operatorname{Ker}(\widetilde{Q})$ if there exists a row $i$ such that $\widetilde{q}_{i j}$ is the unique nonempty element in the $i$-th row. The component $x_{j}$ for all $j \in \operatorname{Ker}(\widetilde{Q})$ is called superattainable.

Remark 5.1 It is easy to see that a column $j_{0} \in$ $\operatorname{Ker}(\widetilde{Q})$ if and only if there exists a row which is covered only by the column $j_{0}$, i.e. the column $j_{0}$ belongs to each covering of $\widetilde{Q}$. Consequently, $\operatorname{Ker}(\widetilde{Q})$ and the row s covered by $\operatorname{Ker}(\widetilde{Q})$ can be removed to reduced the sized of $\widetilde{Q}$.

Let $I_{j}=\left\{i \in I: \widetilde{q}_{i j}\right.$ is the unique nonempty element in row $i$ of $\widetilde{Q}\}$ for all $j \in J$. It is obvious that $I_{j} \neq \emptyset$ if and only if $j \in \operatorname{Ker}(\widetilde{Q})$. For the $i$-th equation $\left(i \in I_{j}, j \in \operatorname{Ker}(\widetilde{Q})\right)$, we have $\mathcal{I}\left(a_{i j}, x_{j}\right)=b_{i}$ since $\widetilde{q}_{i j}$ is a unique nonempty element in row $i$ for each $i \in I_{j}$. Now define $X^{b}=\left(x_{j}^{b}\right)_{j \in J}$ with

$$
x_{j}^{\mathrm{b}}= \begin{cases}\wedge_{i \in I_{j}} \mathcal{I}_{\mathcal{I}}\left(a_{i j}, b_{i}\right), & \text { if } j \in J \text { and } I_{j} \neq \emptyset, \\ 1, & \text { otherwise. }\end{cases}
$$


Remark 5.2 It is straightforward that $X_{*} \leqslant X \leqslant$ $X^{\mathrm{b}}$ for any $X \in \mathscr{X}$ since $X^{\mathrm{b}}$ contains the upper bound to meet all equations corresponding to the elements of $\cup_{j \in J} I_{j}$.

\section{Theorem 5.1 Let system (2) be solvable. Then}

1. System (2) has a unique maximal solution if and only if $X^{b} \in \mathscr{X}$.

2. System (2) has a unique solution if and only if $X^{\mathrm{b}}=X_{*}$.

Proof. (i) Assume that $X^{b} \in \mathscr{X}$, then $X^{b}$ is the unique maximal solution since $X_{*} \leqslant X \leqslant X^{b}$ by Remark 5.2.

Conversely, if system (2) has a unique maximal solution $X^{0}=\left(x_{j}^{0}\right)_{j \in J}$ but $X^{b} \notin \mathscr{X}$, there must exists a row $i_{0}$ of $\widetilde{Q}$ such that it contains more than one nonempty elements and any covering of the row $i_{0}$ can not cover any other rows. Denote $J_{i_{0}}=\{j \in$ $\left.J: \widetilde{q}_{i_{0} j} \neq \emptyset\right\}$. Define $X(j)=\left(x_{k}^{j}\right)_{k \in J}$ for each $j \in J_{i_{0}}$ with

$$
x_{k}^{j}= \begin{cases}x_{k}^{b}, & \text { if } k=j, \\ x_{k}^{0}, & \text { otherwise. }\end{cases}
$$

Obviously, $X(j) \in \mathscr{X}$. Since $X^{0}$ is the unique maximal solution, therefore, $x_{j}^{0}=x_{j}^{b}$ for all $j \in J_{i_{0}}$. However, we have that the elements of row $i_{0}$ in the binary matrix induced by $X^{0}$ are all zeros. By Theorem 3.5, we have $X^{0} \notin \mathscr{X}$, a contradiction.

(ii) It is straightforward from (i).

From Theorem 5.1, Definitions 4.1 and 5.1, the following result holds.

Theorem 5.2 Let $\mathcal{I}$ satisfy the right conditional cancelation law and $\widetilde{Q}$ be the characteristic matrix of system (2). Then

(i) System (2) has a unique solution if and only if all components are supper-attainable.

(ii) System (2) has a unique maximal solution if and only if $\operatorname{Ker}(\widetilde{Q})$ is a unique irredundant covering of $\widetilde{Q}$.

Remark 5.3 1. If all the nonempty elements of $\widetilde{Q}$ happen to be singletons, the construction of $X^{b}$ can be simplified by $X^{b}=\left(x_{j}^{b}\right)_{j \in J}$ with

$$
x_{j}^{b}= \begin{cases}x_{j *}, & \text { if } j \in \operatorname{Ker}(\widetilde{Q}), \\ 1, & \text { otherwise. }\end{cases}
$$

2. If system (2) has a unique solution, then the number of unknowns is no more than the number of equations in system (2), i.e. $n \leqslant m$.

3. System (2) has a unique solution if and only if $\operatorname{Ker}(\widetilde{Q})$ is a unique covering of $\widetilde{Q}$.

Example 5.1 Consider the system $A \circ_{\mathcal{I}_{\mathcal{T}_{P}}} X=B$ with

$$
A=\left(\begin{array}{cccc}
0.3 & 0.8 & 0.1 & 0.2 \\
0.9 & 0.3 & 0.4 & 0.5 \\
0.8 & 0.5 & 0.6 & 0.9 \\
0.6 & 0.3 & 0.8 & 0.2
\end{array}\right) \text { and } B=\left(\begin{array}{l}
0.6 \\
0.7 \\
0.4 \\
0.5
\end{array}\right)
$$

The system is solvable since $X_{*}=$ $(0.63,0.48,0.40,0.36)^{\prime} \quad$ is its smallest solution. The characteristic matrix is $\widetilde{Q}=\left(\begin{array}{cccc}\emptyset & 0.48 & \emptyset & \emptyset \\ 0.63 & \emptyset & \emptyset & \emptyset \\ \emptyset & \emptyset & \emptyset & 0.36 \\ \emptyset & \emptyset & 0.40 & \emptyset\end{array}\right)$. Note that all the nonempty elements of $\widetilde{Q}$ are singletons. Therefore, $\operatorname{Ker}(\widetilde{Q})=\{1,2,3,4\}$. Furthermore, $x_{1}, x_{2}, x_{3}, x_{4}$ are all supper-attainable and $X=(0.63,0.48,0.40,0.36)^{\prime}$ is the unique solution of the system.

Example 5.2 Consider the system $A \circ_{\mathcal{I}_{\mathcal{T}_{L}}} X=B$ with

$$
A=\left(\begin{array}{llll}
0.5 & 0.2 & 0.3 & 0.5 \\
0.6 & 0.5 & 0.2 & 0.9 \\
0.9 & 0.6 & 0.9 & 0.1 \\
0.4 & 0.7 & 0.7 & 0.3
\end{array}\right) \text { and } B=\left(\begin{array}{c}
1 \\
0.7 \\
0.5 \\
0.6
\end{array}\right)
$$

The system is solvable since $X_{*}=(0.5,0.3,0.4,0.6)^{\prime}$ is its smallest solution. The characteristic matrix is $\widetilde{Q}=\left(\begin{array}{cccc}{[0.5,1]} & \emptyset & \emptyset & \emptyset \\ \emptyset & \emptyset & \emptyset & 0.6 \\ \emptyset & \emptyset & 0.4 & \emptyset \\ \emptyset & 0.3 & \emptyset & \emptyset\end{array}\right)$. Obviously, $\operatorname{Ker}(\widetilde{Q})=\{1,2,3,4\}$ and $X^{b}=(1,0.3,0.4,0.6)^{\prime}$ is the unique maximal solution of the system. Although all the columns of $\widetilde{Q}$ are in $\operatorname{Ker}(\widetilde{Q})$, the solutions of the system are not unique in view of the first column of $\widetilde{Q}$ is non-singleton.

\section{Acknowledgements}

This was supported by National Natural Science Foundation of China (Nos. 11201325, 11171242 and 11401410).

\section{References}

[1] M. Baczyński, B. Jayaram, Fuzzy Implications, Studies in Fuzziness and Soft Computing Series, Vol. 231, Springer-Verlag, Heidelberg, 2008.

[2] R. Belohlavek, Sup-t-norm and inf-residuum are one type of relational product: Unifying framework and consequences, Fuzzy Sets and Systems 197(2012) 45-58.

[3] L. Chen, P. P. Wang, Fuzzy relation equations (I): the general and specialized solving algorithms, Soft Computing 6 (2002) 428-435.

[4] B. De Baets, Analytical solution methods for fuzzy relational equations, in: D. Dubois, H. Prade (Eds.), The Handbooks of Fuzzy Sets Series, Vol. 1, Kluwer Academic Publishers, Dordrecht, 2000, pp. 291-340.

[5] F. Di Martino, S. Sessa, Spatial Analysis and Fuzzy Relation Equations, Advances in Fuzzy Systems, Volume 2011, Article ID 429498, 14 pages, 2011. 
[6] A. Di Nola, W. Pedrycz, S. Sessa, Fuzzy relation equations and algorithms of inference mechanism in expert systems, in: Approximate Reasoning in Expert Systems (M. M. Gupta, A. Kandel, W. Bandler and J. B. Kiszka, Eds.), Elsevier Science Publishers B. V. (North Holland), Amsterdam, 1985, pp. 355-367.

[7] A. Di Nola, S. Sessa, W. Pedrycz, E. Sanchez, Fuzzy relation equations and their applications to knowledge engineering, Kluwer Academic Publishers, Dordrecht, 1989.

[8] J. C. Fodor, On contrapositive symmetry of implications in fuzzy logic, In: Proc. 1st European Congress on Fuzzy and Inteligent Technologies (EUFIT 1993), pp. 1342-1348.

[9] J. A. Goguen, The logic of inexact concepts, Synthese 19 (1969) 325-373.

[10] S. C. Han, H. X. Li, J. Y. Wang, Resolution of finite fuzzy relation equations based on strong pseudo-t-norms, Applied Mathematics Letters 19 (2006) 752-757.

[11] C. F. Hu, S. C. Fang, Set covering-based surrogate approach for solving sup- $T$ equation constrained optimization problems, Fuzzy Optimization and Decision Making, 10 (2011) 125152.

[12] H. Imai, K. Kikuchi, M. Miyakoshi, Unattainable solutions of a fuzzy relation equation, Fuzzy Sets and Systems 99 (1998) 193-196.

[13] E. P. Klement, R. Mesiar, E. Pap, Triangular Norms, Kluwer Academic Publishers, Dordrecht, 2000.

[14] P. K. Li, S. C. Fang, On the resolution and optimization of a system of fuzzy relational equations with sup- $T$ composition, Fuzzy Optimization and Decision Making, 7 (2008) 169214.

[15] Y. M. Li, X. P. Wang, Necessary and sufficient conditions for existence of maximal solutions for inf- $\alpha$ composite fuzzy relational equations, Computers and Mathematics with Applications 55 (2008) 1961-1973.

[16] Y. M. Li, X. P. Wang, The solution sets of @-Fuzzy relational equations in finite domains and on a complete Brouwerian lattice. Indian Journal of Pure \& Applied Mathematics 34 (2003) 1249-1257.

[17] J. L. Lin, On the relation between fuzzy maxArchimedean t-norm relational equations and the covering problem, Fuzzy Sets and Systems 160 (2009) 2328-2344.

[18] J. L. Lin, Y. K. Wu, S. M. Guu, On fuzzy relational equations and the covering problem, Information Sciences 181 (2011) 2951-2963.

[19] V. Loia, S. Sessa, Fuzzy relation equations for coding/decoding processes of images and videos. Information Sciences 171 (2005) 145-172.

[20] K. Peeva, Fuzzy linear systems, Fuzzy Sets and Systems 49 (1992), 339-355.

[21] K. Peeva, Y. Kyosev, Algorithm for solving max-product fuzzy relational equations, Soft Computing 11 (2007), 593“C605.

[22] I. Perfilieva, L. Nosková, System of fuzzy relation equations with inf- $\rightarrow$ composition: Complete set of solutions, Fuzzy Sets and Systems 159 (2008) 2256-2271.

[23] A. V. Markovskii, On the relation between equations with max-product composition and the covering problem, Fuzzy Sets and Systems 153 (2005) 261-273.

[24] H. Nobuhara, W. Pedrycz, S. Sessa, Hirota K., A motion compression/reconstruction method based on max t-norm composite fuzzy relational equations, Information Sciences 176 (2006) 2526-2552.

[25] E. Sanchez, Resolution of composite fuzzy relation equations, Information and Control 30 (1976) 38-48.

[26] B. S. Shieh, Solution to the covering problem, Information Sciences 222 (2013) 626-633.

[27] Z. D. Wang, Y. D. Yu, Pseudo-t-norms and implication operations on a complete Brouwerian lattice. Fuzzy Sets and Systems 132 (2002) 113-124.

[28] Z. D. Wang, F. M. Dai, The sets of solutions of $L$-relation equations $\mathcal{T}(a, x)=b$ and $\mathcal{I}(a, x)=$ b. Journal of Yangzhou University 6 (2003) 8-10 (in Chinese).

[29] W. M. Wu, The inf- $\alpha$ compositioin of fuzzy relations, Journal of Shanghai Normal University (Natural). 2 (1985) 1-7 (in Chinese).

[30] Q. Q. Xiong, X. P. Wang, Solution sets of inf- $\alpha_{\mathcal{T}}$ fuzzy relational equations on complete Brouwerian lattices, Information Sciences 177 (2007) 4757-4767.

[31] R. R. Yager, An approach to inference in approximate reasoning, Internat. J. ManMachine Studies 13 (1980) 323-338. 